\title{
Infección por COVID-19, una mirada a los factores ambientales relacionados con la pandemia
}

\author{
COVID-19 infection, a look at environmental factors related to the pandemic
}

Sonia Marcela Rosas Arango ${ }^{1}$, Javier Del Ángel-Caraza², Edgardo Soriano-Vargas ${ }^{3}$

\section{Resumen}

La pandemia global por COVID-19 ha generado un sin número de alertas y cambios en las formas de habitar y de consumo. La triada medio ambiente-salud-individuo vuelve a ser protagonista en este escenario, en donde se aprecian graves fracturas en los sistemas de salud pública, hábitat y seguridad alimentaria. Como factor transversal a esos elementos, la contaminación ambiental se convirtió en un efecto facilitador de la pandemia pues la transmisión por contacto entre humanos a través de aerosoles representa la vía de contagio. Esta situación obligó al distanciamiento y confinamiento preventivo, medida que logró hacer evidente la pobreza, la inequidad y la desigualdad que se vive globalmente. Por otro lado, los cambios en la movilidad y la frecuencia de producción en industrias de manufactura trajo una reducción en los gases de efecto invernadero.

Palabras claves: COVID-19, ambiente, contaminación del aire, cambio ambiental, seguridad alimentaria.

\footnotetext{
1. Docente gestor en investigación, Universidad Santo Tomás.

Correo electrónico: soniarosas@usantotomas.edu.co

ORCID: https://orcid.org/0000-0002-9847-5447

Google Scholar https://scholar.google.es/citations?user=16gZ9BoAAAAJ\&hl=es

2. Médico veterinario, Hospital Veterinario para Pequeña Especies FMVZ-Universidad Autónoma del Estado de México.

Correo electrónico: delangelvet@hotmail.com

ORCID: https://orcid.org/0000-0002-7438-4743

Google scholar https://scholar.google.com/citations?user=MFaxz-cAAAAJ\&hl=de

3. Profesor investigador, Universidad Autónoma del Estado de México.

Correo electrónico: soriano@uaemex.mx

ORCID: https://orcid.org/0000-0002-1514-1741

Google scholar https://scholar.google.com.mx/citations?user=QAa0Krw37Q8C\&hl=es

Profesores investigadores grupo Enfermedades Crónicas y Zoonótica ECZA UCMC
}

https://doi.org/10.22490/24629448.4193 


\section{Abstract}

The global pandemic due to COVID-19 has generated countless alerts and changes in the ways of living and consuming, the environment, health and individual triad is once again the protagonist in this scenario where serious fractures in the public health systems, habitat and food security are seen. As a transverse factor to these elements, environmental contamination became a facilitating effect of the pandemic and transmission by contact between humans through aerosols represents the route of contagion, this situation forced the distancing and preventive confinement, this measure to makes evident the poverty, inequality and inequity that is experienced globally, on the other hand the changes in mobility and the frequency of production in manufacturing industries brought a reduction in greenhouse gases.

Keywords: COVID-19, Air Pollutants,Environmental Change,Food Supply.

La infección por SARS-CoV 2, denominada por la Organización Mundial de la Salud (OMS) como COVID-19 en febrero de 2020 y declarada posteriormente pandemia (1), está, sin duda, asociada a un ciclo que parece no tener límite frente a la susceptibilidad para infecciones respiratorias y la contaminación ambiental. La exposición permanente al material particulado, especialmente el denominado $\mathrm{PM}_{2.5}$, compromete el estado de salud respiratorio y debilita la inmunidad del tracto, lo que promueve la exposición a partículas infecciosas y estimula el estrés oxidativo que impide la rápida recuperación en las enfermedades respiratorias (2). Estas condiciones se convierten en una amenaza permanente frente a los picos epidemiológicos de enfermedad respiratoria, que se rigen principalmente por los cambios estacionales alrededor del mundo.
La infección por SARS-CoV-2 tuvo origen en la ciudad de Wuhan, ubicada en la región central de China en la provincia de Hubei. Es una región industrializada y que genera emisiones permanentes de $\mathrm{CO}_{2} \mathrm{y}$ $\mathrm{NO}_{2}$, situación que se generaliza en el país con el mayor aporte de emisiones de gases invernadero, ligado principalmente a las formas de producción de energía (3).

En 2018, China participó del total de emisiones de $\mathrm{CO}_{2}$ en el planeta con un $28 \%$, seguido de Estados Unidos con un $15 \%$. La variación positiva entre el ańo $2017 \mathrm{y}$ 2018 en China fue de $+2,3 \%$ mientras que para Estados Unidos fue de $+2,8 \%$ (3). Lo anterior indiscutiblemente eleva la susceptibilidad de los habitantes de estos países a padecer enfermedades respiratorias y expone a la población mundial a desarrollar patologías crónicas que resultan importan- 
tes en la morbimortalidad para la infección por SARS-CoV- 2.

La relación entre el material particulado presente en las emisiones atmosféricas y el desarrollo de enfermedades crónicas ha sido documentada. Gorr (4), en 2017, describe la importancia epidemiológica del $\mathrm{PM}_{2,5}$ asociado a enfermedad respiratoria y cardiaca principalmente porque la densidad de la partícula hace que se aloje en el alveolo pulmonar y se distribuya en el torrente sanguíneo. Adicionalmente un aumento en promedio de $10 \mu \mathrm{g} / \mathrm{m}^{3}$ de exposición en la calidad del aire al $\mathrm{PM}_{2,5}$ incrementa el riesgo de muerte por afección cardiaca y genera mayor predisposición a sufrir al menos un evento cardiovascular. $(12,16)$

En la infección por COVID-19 se ha identificado que padecer algún, tipo de enfermedad cardiovascular representa un factor de riesgo para el pronóstico de la infección, debido a que favorece el desarrollo de un síndrome multisistémico fatal $(1,5)$

Las condiciones naturales del comportamiento atmosférico y sus transformaciones asociadas al cambio global juegan un rol significativo en la pandemia; las relaciones entre la humedad, la temperatura y la supervivencia en superficies que favorecen la presencia del SARS-CoV-2, han sido dilucidadas a lo largo de la experiencia de la pandemia. En cuanto a la viabilidad en superficies, el virus sobrevive a temperaturas de hasta $4^{\circ} \mathrm{C},(7,8,9)$ y es estable en un rango de $\mathrm{pH}$ de 3 a 10 en temperatura ambiente (9).

Asimismo, no existe una validación estadísticamente significativa en la relación de la humedad del ambiente y la velocidad del viento frente a la prevalencia de la enfermedad (7). La principal vía de dispersión ambiental es la directa de personas infectadas, mediante las nanogotas emitidas en aerosoles de origen respiratorio como la tos, el estornudo y la saliva; estos aerosoles pueden llegar a contagiar a una distancia de hasta 2 metros $(7,9)$.

Derivada de esta condición de transmisión, el distanciamiento social se ha abordado con medidas tales como el confinamiento preventivo; sin embargo, en muchos países, incluidos los denominados del primer mundo, las condiciones de hábitat no permiten distanciamientos al interior de las viviendas. Los fenómenos migratorios irregulares generan situaciones de exposición de la población y la necesidad de regulación de la economía de consumo expone a los individuos a no guardar esta medida para satisfacer las necesidades básicas.

Así, las condiciones de migración irregular y cambio del uso de los suelos, bien sea por asentamientos humanos irregulares o por desplazamientos asociados al cambio climático, también ha traído consigo un factor detonante frente a la seguridad alimentaria en la pandemia por COVID-19. 
Aquí se conjugan tres factores: en primer lugar, la disminución de recursos económicos para adquirir productos básicos nutricionales; en segundo lugar, la pérdida de áreas de suelo dedicadas al cultivo, y, en tercer lugar, los mecanismos de transmisión zoonótica derivados del consumo de animales salvajes y desplazamiento de fauna silvestre a zonas periurbanas y urbanas.

De acuerdo con la Organización de las Naciones Unidas para la Alimentación y la Agricultura (FAO), 9,2\% de la población mundial estuvo expuesta, en 2018, a niveles graves de seguridad alimentaria, donde África, Asia y América latina y el Caribe fueron los continentes con algún tipo de inseguridad alimentaria declarada (10). A esto se ańade la malnutrición que favorece no solamente los índices bajos de crecimiento y desarrollo, sino también los problemas de obesidad y enfermedad cardiovascular a nivel global. Ambos extremos resultan favorecer la morbimortalidad por COVID-19 $(6,10)$ no solamente en grupos poblacionales de adultos mayores, sino también en población joven que actualmente se ha considerado en riesgo progresivo frente al riesgo cardiovascular (11).

En este panorama de susceptibilidad global de los individuos, el confinamiento también ha generado efectos positivos indirectos sobre condiciones ambientales frente al cambio climático. Muhammad y Salman (12) señalan que, de acuerdo con los reportes de la NASA y otras agencias internacionales en las regiones con mayor prevalencia como Wuhan, Espańa, Italia y Estados Unidos, los efectos del confinamiento generaron reducciones de hasta el $30 \%$ de emisiones contaminantes, principalmente $\mathrm{CO} 2$ y NO2 (12). Este fenómeno también se asocia a la disminución de la movilidad mundial, el consumo de energía y la inactividad de las industrias de manufactura. Barcelo (13), en un estudio que incluyó las regiones de Italia, España, Francia y Alemania con mayor número de casos de muerte por COVID-19, encontró una correlación positiva entre las concentraciones de NO2 del aire en las regiones y la mortalidad. Así, el $78 \%$ de los casos fatales se ubicaron en cinco regiones en el norte de Italia y el centro de España con concentraciones elevadas de $\mathrm{NO} 2$ en el aire (13).

De esta forma, la pandemia, si bien ha generado efectos positivos representados en la disminución de la contaminación de origen antrópico por causas multifactoriales, también se registrará como una calamidad global que vuelve la atención sobre las formas de habitar, la sobrepoblación, la seguridad alimentaria, la ecología y el desarrollo tecnológico para enfrentar la infecciones por microorganismos. 


\section{Referencias}

1. Zhao M, Wang M, Zhang J, Ye J, Xu Y, Wang Z, et al. Advances in the relationship between coronavirus infection and cardiovascular diseases. Biomedicine \& Pharmacotherapy. 2020;127:110230.

2. Horne BD, Joy EA, Hofmann MG, Gesteland PH, Cannon JB, Lefler JS, et al. Short-Term Elevation of Fine Particulate Matter Air Pollution and Acute Lower Respiratory Infection. American Journal of Respiratory and Critical Care Medicine. 2018;198(6):759-66

3. Friedlingstein P, Jones MW, O'sullivan M, Andrew RM, Hauck J, Peters GP, et al. Global Carbon Budget 2019. Earth System Science Data. 2019;11(4):1783-838.

4. Gorr MW, Falvo MJ, Wold LE. Air Pollution and Other Environmental Modulators of Cardiac Function. Comprehensive Physiology. 2017;1479-95

5. Koton S, Molshatzki N, Yuval, Myers V, Broday DM, Drory Y, et al. Cumulative exposure to particulate matter air pollution and long-term post-myocardial infarction outcomes. Preventive Medicine. 2013;57(4):339-44.

6. D'Ippoliti D, Forastiere F, Ancona C, Agabiti N, Fusco D, Michelozzi P, et al. Air Pollution and Myocardial Infarction in Rome. Epidemiology. 2003;14(5):528-35.

7. Eslami H, Jalili M. The role of environmental factors to transmission of SARS-CoV-2 (COVID-19). AMB Express. 2020;10(1).

8. Abdullahi, Idris Nasir et al. "Exploring the genetics, ecology of SARS-COV-2 and climatic factors as possible control strategies against COVID-19.” Le infezioni in medicina. 2020;28(2):166-173.

9. Chin A, Chu J, Perera M, Hui K, Yen H-L, Chan M, Peiris M, Poon L. Stability of SARS-CoV-2 in different environmental conditions. medRxiv. 2020 doi: 10.1101/2020.03.15.20036673.
10. FAO.La seguridad alimentaria y la nutrición en el mundo en 2018. El estado de la seguridad alimentaria y la nutrición en el mundo El estado de la seguridad alimentaria y la nutrición en el mundo 2018. 2019;1-36.

11. Almonacid C, Hernández E, Rosas M, Triana V, y García N.Y Determinación del riesgo cardiovascular y su asociación con la presencia de dislipemias en universitarios de Bogotá-Colombia. Rev Esp Cardiol. 2016:69 Supl 1:5

12. Muhammad S, Long X, Salman M. COVID-19 pandemic and environmental pollution: A blessing in disguise? Science of The Total Environment. 2020;728:138820.

13. Barcelo D. An environmental and health perspective for COVID-19 outbreak: Meteorology and air quality influence, sewage epidemiology indicator, hospitals disinfection, drug therapies and recommendations. Journal of Environmental Chemical Engineering. 2020;8(4):104006. 